\title{
Near-field optical properties and surface plasmon effects generated by a dielectric hole in a silver-shell nanocylinder pair
}

\author{
Yuan-Fong Chau, ${ }^{1, *}$ Han-Hsuan Yeh, ${ }^{1}$ and Din Ping Tsai ${ }^{2}$ \\ ${ }^{1}$ Department of Electronic Engineering, Ching Yun University, Jung-Li 320, Taiwan \\ ${ }^{2}$ Department of Physics and Center of Nanostorage Research, National Taiwan University, Taipei 10617 Taiwan \\ ${ }^{*}$ Corresponding author: yfc01@ @ cyu.edu.tw
}

Received 28 April 2008; revised 3 August 2008; accepted 13 August 2008; posted 15 September 2008 (Doc. ID 95518); published 13 October 2008

\begin{abstract}
Near-field optical properties and surface plasmon effects in a silver-shell nanocylinder pair with five different dielectric holes (DHs) that interact with a transverse magnetic mode incident plane wave are simulated by use of the finite-element method, which includes the investigation of particle-particle interaction. The proposed structure exhibits a redshifted localized surface plasmon that can be tuned over an extended wavelength range by varying the dielectric constant in DHs and the thickness of the nanocylinder silver shell. The increase in the near-field intensity is attributed to a larger effective size of DH that is filled with a higher refractive medium. (C) 2008 Optical Society of America

OCIS codes: $\quad 240.6680,310.6860,260.3910$.
\end{abstract}

The optical properties of metallic nanostructures exhibit a wide variety of optical phenomena that have made them the center of considerable recent attention. Nanoparticles can be used to guide, enhance, emit, and modify optical fields [1,2]. It is well known that individual nanoparticles can support surface plasmon resonance in the optical wavelength range. Surface plasmons are of interest $[3,4]$ because they lead to large electromagnetic field enhancement near the metal surface, peaking at a resonance wavelength that depends on the size, shape, and local dielectric environment of nanoparticles. Because of the localized field amplification that occurs, excitation of surface plasmons in metal nanoparticles based on current-carrying devices can be expected to lead to new and interesting optoelectronic phenomena and applications.

According to recent experimental research [5], two identical gold nanoparticles were found to have strong anisotropy in scattering spectra for different

0003-6935/08/305557-05\$15.00/0

(C) 2008 Optical Society of America polarization directions. Local field enhancement of linear arrays of identical silver nanoparticles exhibits 5000-fold local field intensity enhancement [6]. A dramatic surface plasmon field is excited at the extremity of a finite chain of gold nanoparticles [7]. These results are closely related to surface-enhanced Raman scattering of molecules adsorbed on metallic nanoparticles $[8,9]$. However, nanoshell effects of metallic nanoparticles are not considered. Nanoshells can be constructed by forming an airhole inside metallic nanocylinders. In recent years, the physical and chemical properties of metallic nanoshells have received special attention [10] because they possess several attractive features that make them interesting as nanoscale optical components. They possess a plasmon-derived tunable optical resonance controlled by the dimensions of an airhole in a nanocylinder and the thickness of a metallic shell and span much of the visible and infrared regions of the optical spectrum [11]. Additionally, nanoshells and other nanoscale metallic structures have been shown to enhance local electromagnetic fields in certain regions near their surfaces at specific wavelengths of light, controlled by nanostructure geometry 
$[2,12]$. This subwavelength structure could provide a tool for the manipulation of light below the diffraction limit.

We simulate the near-field response of a silvershell nanocylinder pair that interacts with a transverse magnetic (TM) incident plane wave by using the finite-element method (FEM), which includes the investigation of particle-particle interaction. The enclosure of a silver-shell nanocylinder pair with a dielectric hole (DH) forms an open cavity model, and the electromagnetic field is effectively confined within the gap of the pair to generate high local field enhancement. The refractive medium in a $\mathrm{DH}$ and the radius of a $\mathrm{DH}$ are the main characteristics that could endow additional variables to explore and tune the near-field optical properties between nanocylinders. We compared the optical response in the nearfield zone of a silver-shell nanocylinder pair with a solid silver one. The influences of wavelength of incident light, interparticle distance, the radii of $\mathrm{DHs}$ and the refractive index in $\mathrm{DHs}$ on local-field enhancement are discussed in our simulations.

The dispersion properties of the metal must be considered here since the absorption and permittivity of the metallic material are frequency dependent. The Drude model is used to describe the dependence of the metallic permittivity on the frequency $[13,14]$. The two-dimensional FEM that we applied to metal nanoparticles was comprehensively described in Refs $[13,14]$. We have used the silver permittivity data obtained from Johnson and Christy [15] and corrected with the Drude model, which includes the size effect [16]. In our formulation we used triangular highorder edge elements. To model an infinite simulation region with a two-dimensional finite-geometry model (i.e., to enclose the computational domain without affecting the numerical solution), it is necessary to use anisotropic perfectly matched layers (PMLs) $[13,14]$ that are placed before the outer boundary. This formulation can be used to deal with anisotropic material in terms of both dielectric permittivity and magnetic permeability, allowing anisotropic PMLs to be implemented directly.

Surface plasmon resonance is usually sensitive to different wavelengths of incident light. With a simple solid silver nanocylinder, strong light absorbing and scattering occur at the plasmon resonance frequency and high local fields are excited around the nanoparticle [17]. However, in a system that consists of a solid silver or a silver-shell nanocylinder pair, the surface plasmon broadens and the resonant condition is modified for particle-particle interaction. It is necessary to investigate the wavelength-dependent responses of a solid silver or a silver-shell nanocylinder pair to understand the roles of the surface plasmon effect and particle-particle interaction. First, the difference of near-field optical response and surface plasmon resonance (SPR) on a solid silver nanocylinder pair [Fig. 1(a)] and a silver-shell nanocylinder pair with a different refractive medium in a DH [Fig. 1(b)] is investigated. We compare the near-

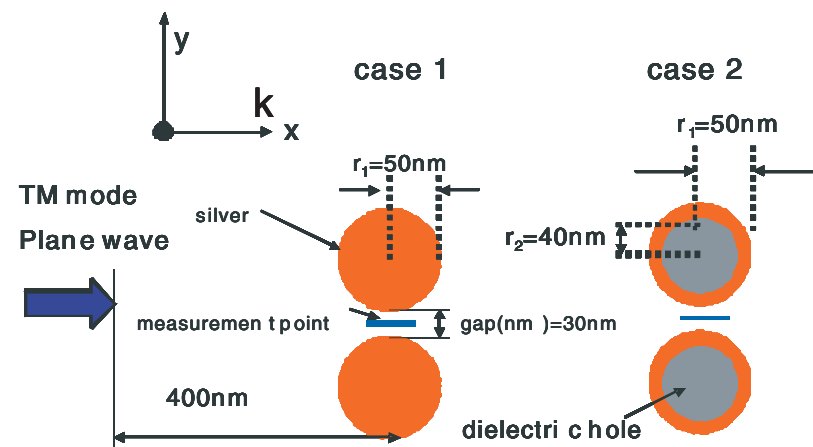

(a)

(b)

Fig. 1. (Color online) Schematic diagram of (a) a solid silver nanocylinder pair and (b) a silver-shell nanocylinder pair.

field optical response of case 1 [see Fig. 1(a), a solid silver nanocylinder pair] with case 2 [see Fig. 1(b), a $\mathrm{DH}$ filled with dielectric constant $\varepsilon=1,1.77,2.31$, 2.66 , and 3.06 [18]], which are illuminated with a TM electromagnetic plane wave and propagated in the $k$ direction. The direction of electric field $E$ is perpendicular to $k$ and parallel to the plane of incidence. The wavelength of incident light varies from 350 to $800 \mu \mathrm{m}$. The gap (interparticle distance) is set to $\mathrm{g}=$ $30 \mathrm{~nm}$ and the near-field intensity is measured in the central part of the gap. Near-field intensities of local fields in the gaps between two nanocylinders are also quite sensitive to the radius of the nanocylinders. On the basis of our simulations, we set the radius at $r_{1}=50 \mathrm{~nm}$ in case 1 , and the outer and inner radii of $r_{1}=50 \mathrm{~nm}$ and $r_{2}=40 \mathrm{~nm}$ in case 2 , respectively. Figure 2(a) shows the simulation results of the nearfield intensities of case 1 (no DH) and case 2 (with five different $\mathrm{DHs}$ ) as a function of wavelengths of TM incident light. We found only one transmission peak in case 1 and more than one peak in case 2 , which corresponds with the SPR mode. This phenomenon can be explained by the combination of SPR and the filling refractive medium in DHs. The holes in nanocylinders can be considered as truncated circular waveguides. The gap (cavity) between the nanoshell is open to the free space and forms a low- $Q$-cavity resonator [19]. The near-field intensities reach peaks at which the Fabry-Perot resonance condition is satisfied [20]. On the basis of our simulations, the value of the near-field intensity depends on the medium filled in DHs, the thickness of the nanoshell, and the gap width. As observed by our simulation results, filling a higher refractive medium in $\mathrm{DHs}$ increases the effective size of the DH and also increases the

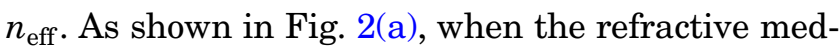
ium filled the DHs, varying from $1,1.77,2.31,2.66$ to 3.06 , the near-field intensity is incremental and the maximum SPR mode (peak) tends toward a higher wavelength. Note that increasing the refractive medium in DHs results in a higher field intensity and a narrower peak width, and a trend to the redshift with the increase of the dielectric constant in $\mathrm{DHs}$. 


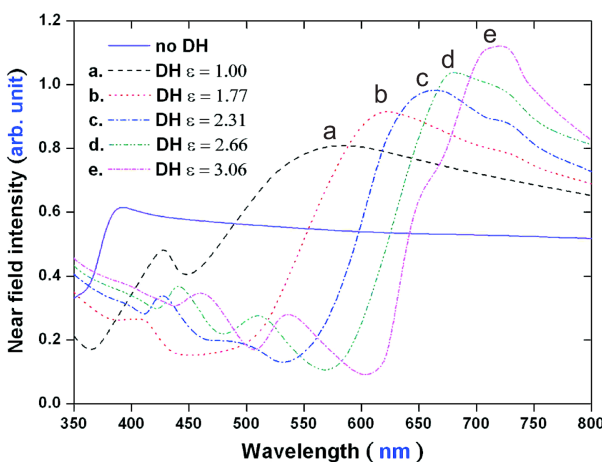

(a)
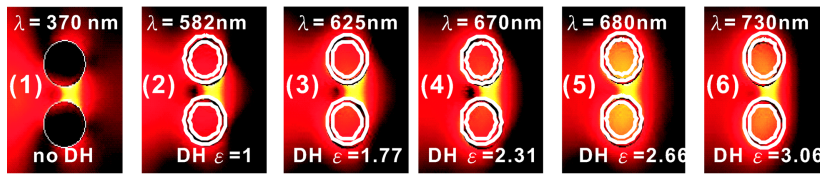

(b)

Fig. 2. (Color online) (a) Near-field intensities in the gaps of cases 1 and 2 as a function of the wavelengths of TM incident light ranging from 350 to $800 \mathrm{~nm}$. (b) The corresponding TM mode near-field distributions in cases 1 and 2 .

The corresponding resonant peak wavelength for case 1 is $370 \mathrm{~nm}$; for case 2 the resonant peak wavelengths are $582 \mathrm{~nm}$ for $\epsilon=1.0,625 \mathrm{~nm}$ for $\epsilon=1.77$, $670 \mathrm{~nm}$ for $\epsilon=2.31,680 \mathrm{~nm}$ for $\epsilon=2.66$, and $730 \mathrm{~nm}$ for $\epsilon=3.06$. The corresponding TM mode near-field distributions in cases 1 and 2 are (1) $\lambda=370 \mathrm{~nm}$ for no DH (case 1), (2) $\lambda=582 \mathrm{~nm}$ for $\epsilon=1$ (airhole), (3) $\lambda=625 \mathrm{~nm}$ for $\epsilon=1.77$, (4) $\lambda=670 \mathrm{~nm}$ for $\epsilon=2.31$, (5) $\lambda=680 \mathrm{~nm}$ for $\epsilon=2.66$, and (6) $\lambda=730 \mathrm{~nm}$ for $\epsilon=$ 3.06 as shown in Fig. 2(b). In case 1, the high localfield enhancements appear in the vicinity of the solid silver nanocylinder pair and light is confined effectively within the gap of the pair due to surface plasmon excitation. The field localization in the gap of a solid silver nanocylinder pair is determined by the polarization direction of the electric field of the incident light. For a single-pair system, the local field vanishes in the gap of the nanocylinder pair when the direction of the incident electric field is perpendicular to the major axis of the pair [21].

Turning to the silver-shell nanocylinder pair filled with a refractive medium in the $\mathrm{DH}$ as shown in case 2 , we have another variable $\varepsilon$ (filling refractive medium in DHs) to explore the near-field optical properties of the silver-shell nanocylinder pair. As can be seen in Fig. 2(b), the strong electromagnetic coupling between the inner and the outer shell walls when the thickness $\left[d=\left(r_{1}-r_{2}\right)\right]$ is small compared to nanocylinder radius $r_{1}$. The geometries are metal-air gap-metal for case 1 and dielectric-metal film-air gap-metal film-dielectric for case 2 . There are many interfaces between the metal and the dielectrics. It is worth noting the structure of the case 2 system whose outer environment of the silver-shell nanocylinder pair is air. Then only one geometry in case 2, which is for $\mathrm{DH} \epsilon=1$, is symmetrical for the thin metal film, i.e., air-Ag-air, and the plasmon mode splitting can occur based on symmetric and antisymmetric mode branches. For the other four situations, $\epsilon>1.0$, the geometry for metal film is nonsymmetrical, i.e., air-Ag-dielectric, there will be only two independent single interface modes and not any mode splitting phenomenon.

The effect of silver-shell thickness on the near-field intensity has also been investigated. Figure 3 shows the simulation results of the near-field intensities in the gaps of case 2 as a function of $\mathrm{DH}$ radius $\left(r_{2}\right)$ at different peak wavelengths of TM incident light. The gap (interparticle distance) is set to $g=30 \mathrm{~nm}$ and the near-field intensity is measured in the central part of the gap. It can be clearly seen in Fig. 3 that the resonance peaks occur near $r_{2}=40 \mathrm{~nm}$ for $\epsilon=1.77,2.31,2.66,3.06$ and near $r_{2}=42 \mathrm{~nm}$ for $\epsilon=$ 1 (airhole case). The performance of the near-field intensity in case 2 is almost the same as $r_{2}$ less than $35 \mathrm{~nm}$ (i.e., thickness of $d>15 \mathrm{~nm}$ ) due to a silvershell thickness $d$ larger than $15 \mathrm{~nm}$, which can be regarded as a solid silver nanocylinder pair. In contrast, the near-field intensities in the gaps of a silver-shell nanocylinder pair decrease rapidly when the radius of $\mathrm{DH} r_{2}$ is increased from 40 to $50 \mathrm{~nm}$ for $\epsilon=1.77,2.31,2.66$, and 3.06 , and from 43 to $50 \mathrm{~nm}$ for $\epsilon=1$ (airhole case). One of the reasons for the decreased intensities in the gaps of case 2 for $\mathrm{DH} \epsilon=$ 1.0 (antisymmetric case) can be attributed to the decreased thickness of the silver shell in nanocylinders, which results in less interaction between the incident light and the thinner silver shell. In principle, at least for the symmetrical geometry of a thin metal film, i.e., dielectric-thin metal filmdielectric, the thinner the metal film, the longer the propagating distance of the long-range surface plasmon mode, the stronger the field enhancement near the interface between the metal and the

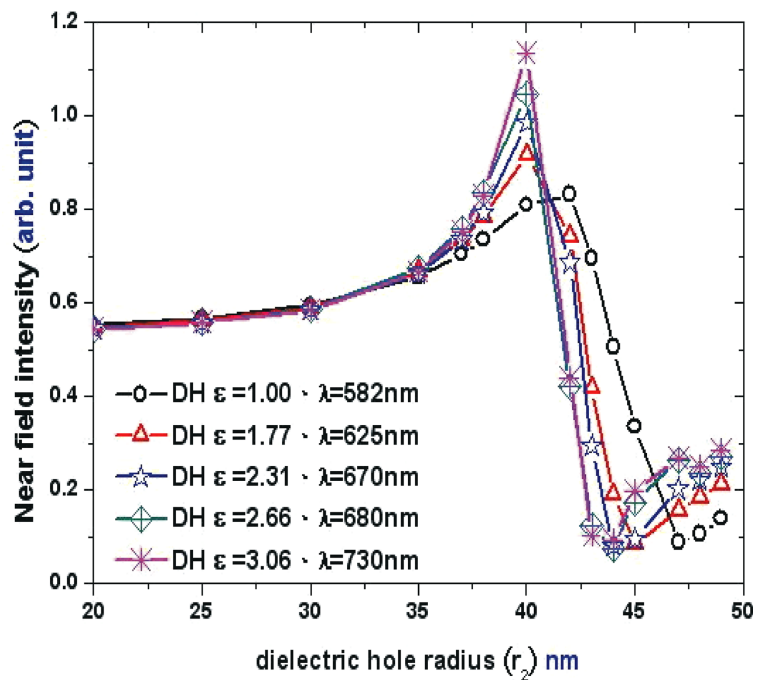

Fig. 3. (Color online) Results of near-field intensity versus DH radii. The parameters are maintained as the outer radius of a silver shell nanocylinder pair $\mathbf{r}_{1}=50 \mathrm{~nm}$, interparticle distance $\mathbf{g}=30 \mathrm{~nm}$, and a DH silver-shell nanocylinder pair with dielectric constants as listed. 
dielectric and also the shorter the penetrating depth through the dielectric. The situation for nonsymmetrical geometry in case 2 for $\mathrm{DH} \epsilon>1.0$ (nonsymmetric case) is more complex; it depends on the amount of nonsymmetry and on how thin the metal film is. Because of the complicated interfaces between metal and dielectrics in case 2 for $\mathrm{DH}$ $\epsilon>1.0$, only the field intensity is measured because the middle position of the gap between the two silvershell nanocylinders is not sufficient to provide complete information for the relationship between the field intensity and the thickness of the metal shells. It is worth noting that the field intensity of the local field in the gaps between the nanocylinders is quite sensitive to the thickness of silver shell and the refractive medium filled DHs. The increase in the near-field intensity is attributed to a larger effective size of the $\mathrm{DH}$ when it is filled with a higher refractive index medium [22].

Figure 4 shows the simulation results of the nearfield intensities in the gaps of the case 2 (with five different $\mathrm{DHs}$ ) system as a function of gap width at different peak wavelengths of TM incident light. The outer and inner radii are $r_{1}=50 \mathrm{~nm}$ and $r_{1}=40 \mathrm{~nm}$, respectively, and the near-field intensity is measured in the central part of the gap. It can be clearly seen from Fig. 4 that the near-field intensity decreases as the gap width and the refractive index of the $\mathrm{DH}$ decrease.

The field distributions can help us to understand how light is distributed through the DHs. To realize the detailed behavior of the field distribution inside a silver-shell nanocylinder pair, Fig. 5 shows the nearfield intensity versus a different refractive index of the $\mathrm{DH}$ at relative peak wavelengths along the $y$ axis (at $x=0$, shown in the Fig. 5 inset) in the range of $y=[-65,65] \mathrm{nm}$. The outer and inner radii are kept

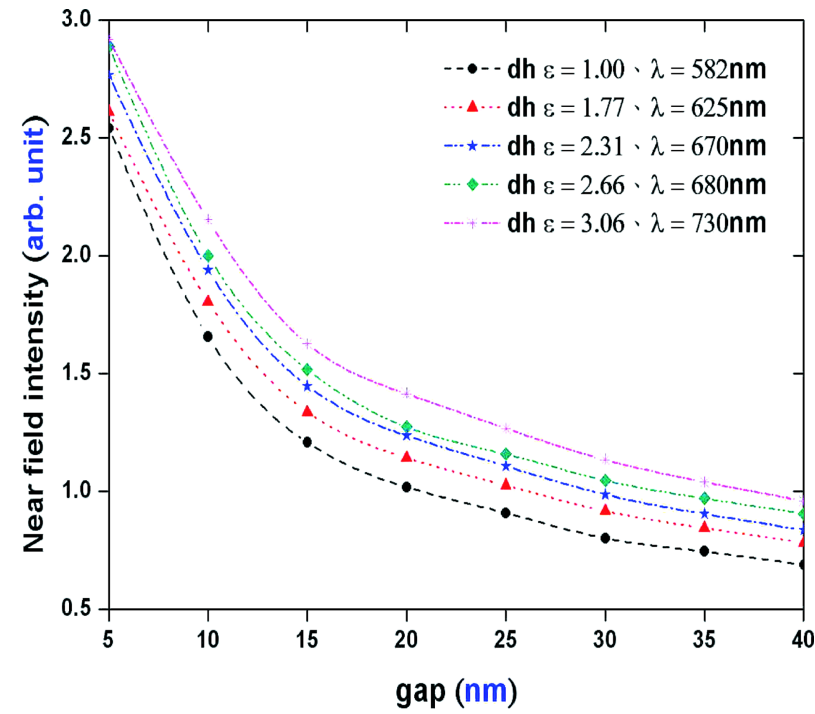

Fig. 4. (Color online) Results of near-field intensity versus gap width. The parameters are maintained as the outer radius of a silver-shell nanocylinder pair $\mathbf{r}_{1}=50 \mathrm{~nm}$, the inner radius of a silver-shell nanocylinder pair $\mathbf{r}_{2}=50 \mathrm{~nm}$, and the $\mathrm{DH}$ silver-shell nanocylinder pair with dielectric constants as listed.

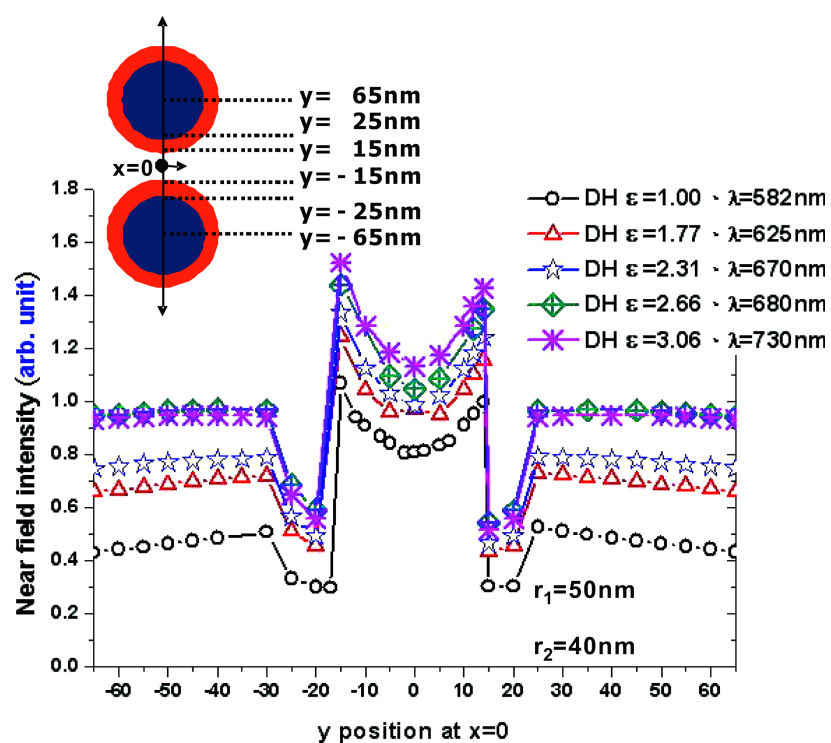

Fig. 5. (Color online) Near-field intensity versus different refractive indices of a $\mathrm{DH}$ at relative peak wavelengths along the $y$ axis (at $x=0$, shown in the Fig. 5 inset) in the range of $y=[-65,65] \mathrm{nm}$. The outer and inner radii of a silver shell nanoscylinder pair are $r_{1}=50 \mathrm{~nm}$ and $r_{2}=40 \mathrm{~nm}$, respectively.

at $r_{1}=50 \mathrm{~nm}$ and $r_{2}=40 \mathrm{~nm}$, respectively. As expected from previous simulations, it can be observed in Fig. 5 that the higher refractive index of $\mathrm{DH}$ results in higher near-field intensities. For the atoms along the circle edges of the silver-shell nanocylinder pair can be considered as many dipoles at the symmetry positions around the circumference of the $\mathrm{DH}$. The TM polarization field intensity pattern can be understood as a strong dipolar excitation that involves the entire ring perimeter, and the equal signs at opposite sides of the nanoshell wall interact to a degree determined by the nanoshell wall thickness [10]. In contrast, the antisymetric branches correspond to patterns of oscillation of opposite sign along both nanoshell walls. The strongest field intensity peak value is found at the circumference of the silver-shell edge of $y=-15$ and $15 \mathrm{~nm}$ as shown in Fig. 5. Note that the localized electric field enhancement at the edge along the silver shell is due to the edge enhancement effect [23]. The field enhancement of the $\mathrm{Ag}$ nanorod originates mainly from the localized surface plasmon mode excited by the evanescent field. These near-field optical phenomena of the metallic nanostructure correspond to the nearfield optical properties that were found in previous simulations and experiments [10].

In summary, we have shown that a silver-shell nanocylinder pair exhibits tunable plasmon resonances in the near-field zone that are not observed for a solid silver nanocylinder pair of similar size. The increase in the near-field intensity is attributed to the larger effective size of the DH filled with the higher refractive medium. The volume confined by the silver-shell nanocylinder pair is filled with refractive medium in $\mathrm{DHs}$ and is, therefore, accessible to various sensing and spectroscopy applications at the nanometer 
scale. As observed from numerical simulations, the main features can be qualitatively understood from a simple silver-shell nanocylinder pair model. Silvershell nanocylinder pairs could serve as resonant nanocavities to hold and probe smaller nanostructures, such as biomolecules or quantum dots. The predictive character of these calculations allows one to tailor the shape of the nanoparticle to achieve excitation spectra on demand with a controlled field enhancement.

The authors are grateful for the financial support from the National Science Council, Taiwan, under grants NSC-96-2120-M-002-017 and NSC 96-2112M-231-001-MY3.

\section{References}

1. Y. W. C. Cao, R. C. Jin, and C. A. Mirkin, "Nanoparticles with Raman spectroscopic fingerprints for DNA and RNA detection," Science 297, 1536-1540 (2002).

2. K. Li, M. I. Stockman, and D. J. Bergman, "Self-similar chain of metal nanospheres as an efficient nanolens," Phys. Rev. Lett. 91, 227402 (2003).

3. W. Dickson, G. A. Wurtz, P. Evans, D. O'Connor, R. Atkinson, R. Pollard, and A. V. Zayats, "Dielectric-loaded plasmonic nanoantenna arrays: a metamaterial with tuneable optical properties," Phys. Rev. B 76, 115411 (2007).

4. R. Atkinson, W. R. Hendren, G. A. Wurtz, W. Dickson, A. V. Zayats, P. Evans, and R. J. Pollard, "Anisotropic optical properties of arrays of gold nanorods embedded in alumina," Phys. Rev. B 73, 235402 (2006).

5. W. Rechberger, A. Hohenau, A. Leitner, J. R. Krenn, B. Lamprecht, and F. R. Aussenegg, "Optical properties of two interacting gold nanoparticles," Opt. Commun. 220, 137-141 (2003).

6. L. A. Sweatlock, S. A. Maier, H. A. Atwater, J. J. Penninkhof, and A. Polman, "Highly confined electromagnetic fields in arrays of strongly coupled Ag nanoparticles," Phys. Rev. B 71, 235408 (2005).

7. P. Ghenuche, R. Quidant, and G. Badenes, "Cumulative plasmon field enhancement in finite metal particle chains," Opt. Lett. 30, 1882-1884 (2005).

8. M. Moskovits, "Surface-enhanced spectroscopy," Rev. Mod. Phys. 57, 783-826 (1985).
9. S. Nie and S. R. Emory, "Probing single molecules and single nanoparticles by surface-enhanced Raman scattering," Science 275, 1101-1106 (1997).

10. J. Aizpurua, P. Hanarp, D. S. Sutherland, M. Käll, G. W.Bryant, and F. J. García de Abajo, "Optical properties of gold nanorings," Phys. Rev. Lett. 90, 057401 (2003).

11. E. Prodan, , P. Nordlander, and N. J. Halas, "Electronic structure and optical properties of gold nanoshells," Nano Lett. 3, 1411-1415 (2003).

12. J. B. Jackson, S. L. Westcott, L. R. Hirsch, J. L. West, and N. J. Halas, "Controlling the surface enhanced Raman effect via the nanoshell geometry," Appl. Phys. Lett. 82, 257 (2003).

13. P. M. Gresho and R. L. Sani, Incompressible Flow and Finite Element Method (Wiley, 2000), Vols. 1 and 2.

14. COMSOL Multiphysics, http://www.comsol.com.

15. P. B. Johnson and R. W. Christy, "Optical constants of the noble metals," Phys. Rev. B 6, 4370-4379 (1972).

16. T. Okamoto, "Near-field spectral analysis of metallic beads." in Near-Field Optics and Surface Plasmon Polaritons, Vol. 81 of Topics in Applied Physics, S. Kawata, M. Ohtsu, and M. Irie, eds. (Springer, 2001), p. 99.

17. Y.-F. Chau and D. P. Tsai, "Three-dimensional analysis of silver nano-particles doping effects on super resolution near-field structure," Opt. Commun. 269, 389-394 (2007).

18. Y. Chen, Y. Wang, Y. Zhang, and S. Liu, "Numerical investigation of the transmission enhancement through subwavelength hole array," Opt. Commun. 274, 236-240 (2007).

19. Z. Ruan and M. Qiu, "Enhanced transmission through periodic arrays of subwavelength holes: the role of localized waveguide resonances," Phys. Rev. Lett. 96, 233901 (2006).

20. Q. Cao and P. Lalanne, "Negative role of surface plasmons in the transmission of metallic gratings with very narrow slits," Phys. Rev. Lett. 88, 057403 (2002).

21. C. Bohren and D. Huffman, Absorption and Scattering of Light by Small Particles (Wiley, 1983).

22. K. L. van der Molen, F. B. Segerink, N. F. van Hulst, and L. Kuipers, "Influence of hole size on the extraordinary transmission through subwavelength hole arrays," Appl. Phys. Lett. 85, 4316-4318 (2004).

23. Y.-F. Chau, T.-J. Yang, and D. P. Tsai, "Imaging properties of three dimensional aperture near-field scanning optical microscopy and optimized near-field fiber probe designs," Jpn. J. Appl. Phys. 43, 8115-8125 (2004). 